\title{
Detection of surface cracks in metals under coatings by induction thermography
}

\author{
by Y. Wang*, X. Gao*, U. Netzelmann** \\ *Nondestructive Testing Research Center, School of Physical Science and Technology, Southwest Jiaotong \\ University, Chengdu, PR China \\ ${ }^{* *}$ Fraunhofer Institute for Nondestructive Testing IZFP, Dept. Components and Assemblies, Campus E3 1, \\ 66123 Saarbrücken, Germany, udo.netzelmann@izfp.fraunhofer.de
}

\begin{abstract}
The effect of non-metallic polymer coatings on the induction thermographic signal from metal surfaces with cracks was studied. Analytical calculations for the backward thermal wave propagation were performed both in the frequency and in the time domain. Numerical simulations were used to show the thermal patterns of cracks under coatings. Experiments were performed on coated samples with artificial cracks. The crack contrast was analysed under coatings of various thickness. The contrast in amplitude and phase is decreasing with coating thickness. The effect of infrared transparency of a model coating was shown in experiment.
\end{abstract}

\section{Introduction}

Induction thermography is a powerful technique to detect surface cracks in metals. Eddy currents cause heating of the metal and cracks generate additional contrast due to changes in the locally induced current density. This can be recorded by an infrared camera. In some cases, the surface to be tested is covered by a coating. This may have a positive effect by increasing surface emissivity and surface homogeneity. On the other hand, it delays and damps the thermal pulse or wave on its way to the coating surface. It has been shown previously that surface cracks in turbine blades can be detected under ceramic thermal barrier coatings [1,2]. Other studies were focused on detection of cracks under corrosion layers on steel [3] or the detection of corrosion under paint layers [4]. Recently, cracks in steel were detected under a glued CFRP layer [5]. Polymer paint coatings on steel with surface cracks protection were less investigated. A quantitative theory for the coating effect is missing. Aim of this contribution is to describe the effect of such electrically non-conducting coatings in analytical theory, simulation and experiment.

\section{One-dimensional analytical models}

In induction thermography, the temperature contrast of a crack is related to the contrast of its undisturbed surroundings. The absolute of the relative temperature contrast usually does not exceed a value of one [6]. Therefore, discussion of the temperature contrast from the sound areas under a coating already provides some insight to the possible defect contrast. Starting point of the analysis is the effect of a coating on a surface heated thermally thick metal on the one-dimensional heat flow. Calculations were performed both in the frequency domain for modulated excitation and in the time domain for pulsed excitation.

\subsection{Frequency domain analysis}

For the frequency domain model, a surface heat source at the metal-coating interface was assumed (figure 1). This can be justified as the typical skin depth of ferritic steel is only about $50 \mu \mathrm{m}$ at $100 \mathrm{kHz}$. The characteristic length for resistive eddy current heating is then even only $25 \mu \mathrm{m}$, which can be considered as small compared to the relevant thermal diffusion lengths. At the same time, the steel substrate is assumed to be thermally thick. Then, the onedimensional diffusion equation can be solved by considering thermal waves as sketched in Figure 1 . The thermal diffusion equation can be solved by using the boundary conditions of the steadiness of the temperature and of the heat flow at the two interfaces. The heat input $q$ is assumed to be due to a plane source with periodically released power density at the coating-metal interface $(x=0)$. 


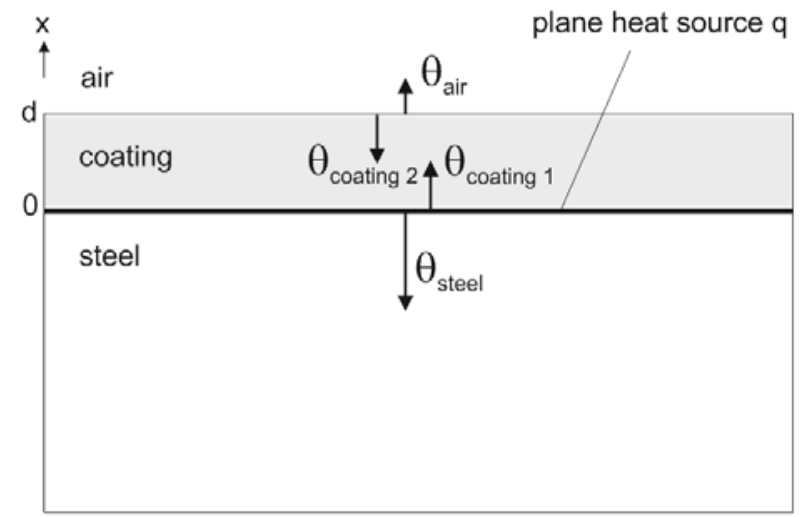

Fig. 1: Sketch of the thermal wave model considered.

After solving the equations for the thermal waves, the temperature oscillation $\theta$ at the polymer surface (at $x=d)$ can be calculated as

$$
\theta\left(\omega_{m}\right)=\frac{2 q}{e_{s} \sqrt{\omega_{m}}} e^{-i \frac{\pi}{4}} \frac{1}{(g b+1)\left(e^{\sigma_{c} d}+e^{-\sigma_{c} d}\right)+(b+g)\left(e^{\sigma_{c} d}-e^{-\sigma_{c} d}\right)} \text {, with } \sigma_{c}=\frac{(1+i)}{\sqrt{2}} \sqrt{\frac{\omega_{m}}{\alpha_{c}}}=\frac{(1+i)}{\mu_{c}}
$$

Here, $g$ and $b$ are the effusivity ratios of air and coating or of coating and metal, respectively. $e_{s}$ is the effusivity of the metal, $\alpha_{c}$ the thermal diffusivity of the coating, $\omega_{\mathrm{m}}$ is the modulation frequency, and $\mu_{\mathrm{c}}$ the thermal diffusion length of the coating.

The terms $\exp \left(\sigma_{c} d\right)$ in equation (1) will be dominant for larger coating thickness. This will lead to exponential damping of the surface temperature oscillation with the thermal diffusion length of the coating as a characteristic length. This is further illustrated in figure 2, where the temperature amplitude and phase at the polymer coating surface based on Eq. (1) are plotted as a function of the coating thickness $d$ for a typical polymer-steel system. The modulation frequency was $50 \mathrm{~Hz}$, the thermal diffusion length of the coating $33.4 \mu \mathrm{m}$.
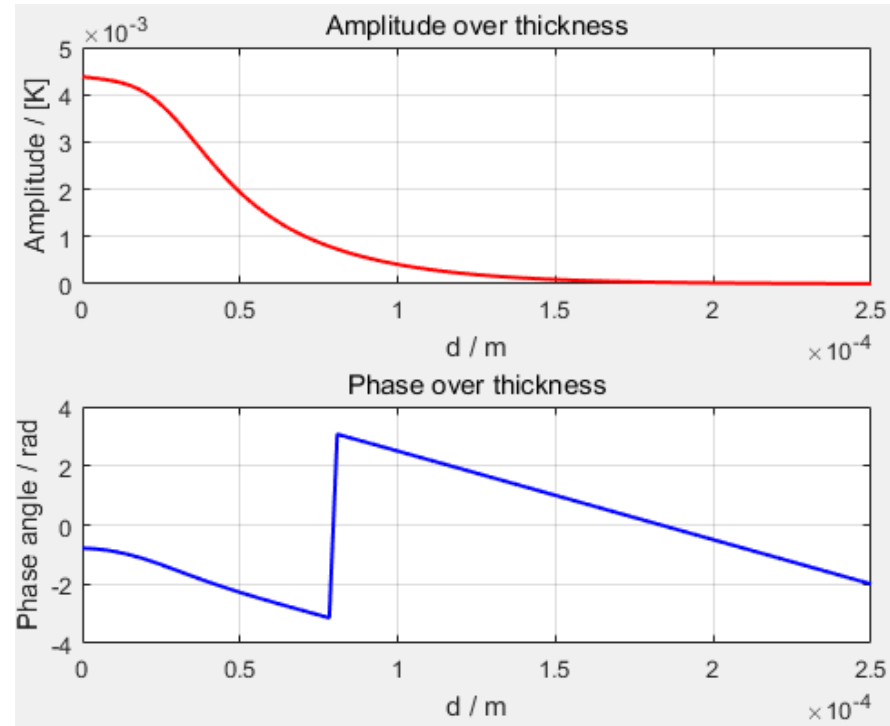

Fig. 2: Calculated surface temperature amplitude (top) and phase (bottom) as a function of the coating thickness

The thermal amplitude decreases rapidly, when the thickness exceeds the thermal diffusion length. The signal phase is decreasing monotonously with thickness, which represents the time delay effect of the coating. 


\subsection{Time-domain analysis}

In the time domain, the starting point is a Green function $G(x, t)$ for a plane instantaneous heat source in the volume at $\mathrm{x}=0$ with an energy density $\mathrm{Q}$. In the coordinates of figure 1 it can be written as:

$$
G(x, t)=\frac{Q}{\rho c} \frac{1}{2 \sqrt{\pi \alpha t}} e^{-\frac{x^{2}}{4 \alpha t}}
$$

When the heat is generated at the boundary of two materials with effusivities $e_{s}$ and $e_{c}$, the boundary condition of steadiness of the temperature at the boundary requires that the energy is splitted into two parts.

$$
Q_{c}=Q \frac{e_{c}}{e_{c}+e_{s}} \text { and } Q_{s}=Q \frac{e_{s}}{e_{c}+e_{s}}
$$

where the first part drives the thermal pulse into the coating, the second one the thermal pulse into the substrate. For simplification, in the following the interface between coating and air is assumed to be adiabatic. The thermal reflection factor at the coating-air interface is then +1 . The method of images can be used to calculate the temperature change at the coating surface by considering the multiple internal reflections in the coating. If

$$
R=\frac{e_{c}-e_{s}}{e_{c}+e_{s}}
$$

is the thermal reflection factor at the interface polymer-steel, the surface temperature change $\Theta(d, t)$ can be written as:

$$
\theta(d, t)=\frac{Q}{\rho_{c} c_{c}} \frac{e_{c}}{e_{c}+e_{s}} \frac{1}{\sqrt{\pi \alpha_{c} t}}\left[e^{-\frac{d^{2}}{4 \alpha_{c} t}}+\sum_{n=1}^{\infty} R^{n} e^{-\frac{(2 n+1)^{2} d^{2}}{4 \alpha_{c} t}}\right]
$$

Eq. 5 was visualized for a polymer coating on steel. In Figure 3 the surface temperature on the polymer surface is shown as a function of the time after delta pulse heating. The temperature response was calculated for three thicknesses of the coating. The delay and the damping of the peak temperature change are clearly visible.

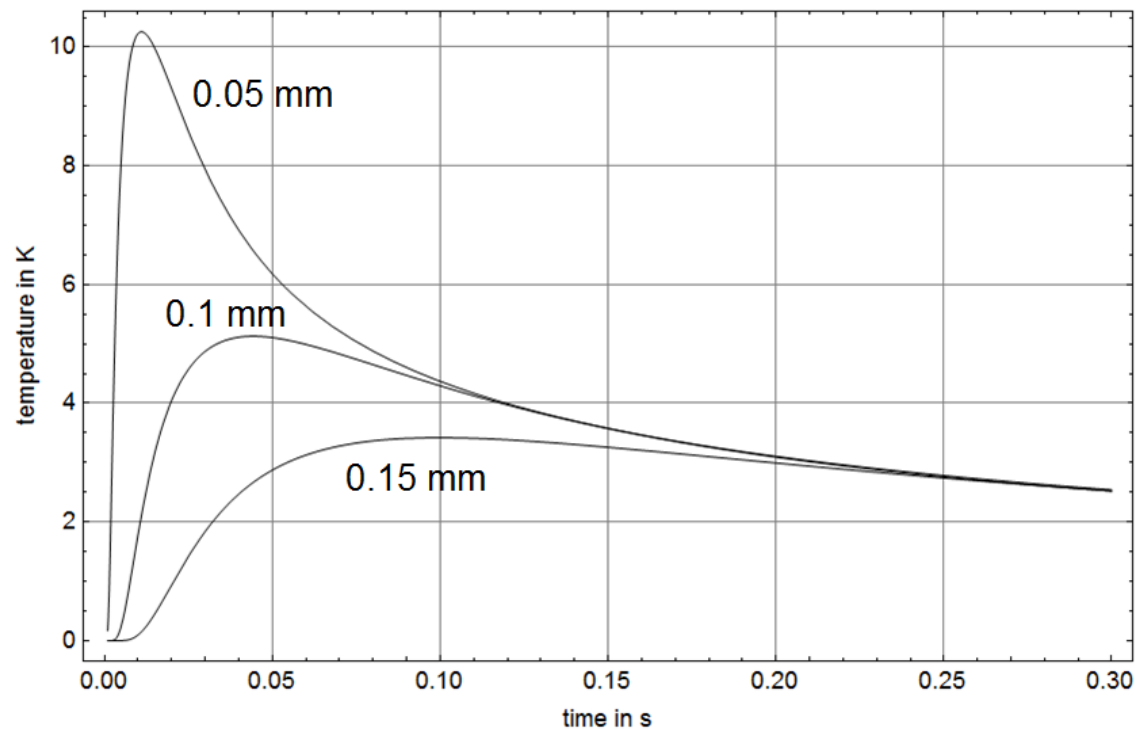

Fig. 3: Calculated surface temperature change on a polymer coating on steel. The coating thickness $d$ was $0.05 \mathrm{~mm}, 0.1 \mathrm{~mm}$ and $0.15 \mathrm{~mm}$, respectively. 


\section{Numerical simulations of the crack contrast}

As the 2D and 3D effects of cracks are difficult to handle by analytical modelling, numerical simulations for the signal of cracks were performed by 2D and 3D numerical simulations using COMSOL Multiphysics 5.3.

\subsection{D calculation}

Figure 1 shows a series of cross-sections through of a coated sample $0.2 \mathrm{~s}$ after begin of heating for different coating thickness. The crack in the ferritic steel was $2 \mathrm{~mm}$ deep, infinitely long, $0.2 \mathrm{~mm}$ wide and filled with air. The coating has typical material parameters of a polymer. The induction frequency was $100 \mathrm{kHz}$. It can be seen how the surface temperature profile over the crack is influenced by the coating. The thicker the coating, the smaller is the temperature contrast.
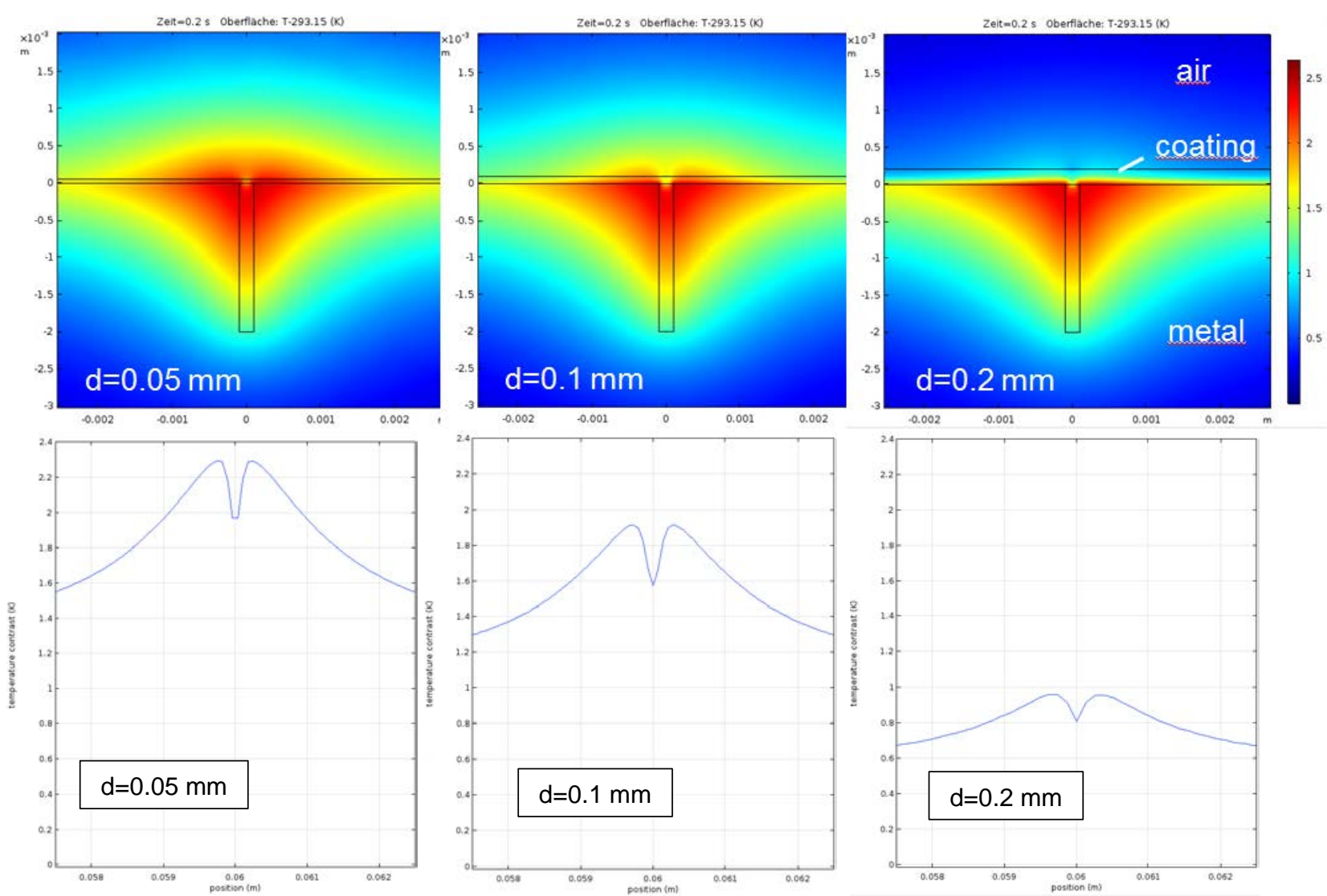

Fig. 4: Top row: Calculated temperature distributions in the cross-section of a polymer coated sample with a long crack in ferritic steel with varying coating thickness $d$. Bottom row: Corresponding plots of the temperature contrast at the coating surface

\subsection{D calculation}

A 3D calculation of a perpendicular crack in ferritic steel was performed assuming a wire as inductor which was crossing the crack at half of its length. The crack was $10 \mathrm{~mm}$ long, $3 \mathrm{~mm}$ deep and $0.5 \mathrm{~mm}$ wide. The wire had a diameter of $2 \mathrm{~mm}$ and was $3 \mathrm{~mm}$ above the metal surface. The induction frequency was $100 \mathrm{kHz}$. 


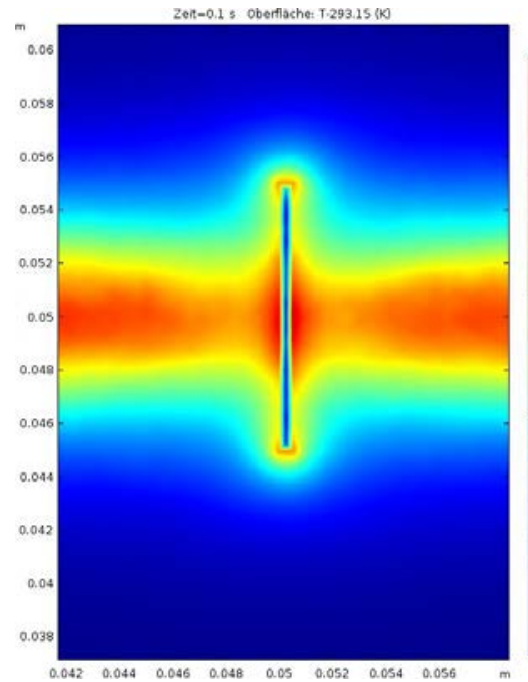

a)

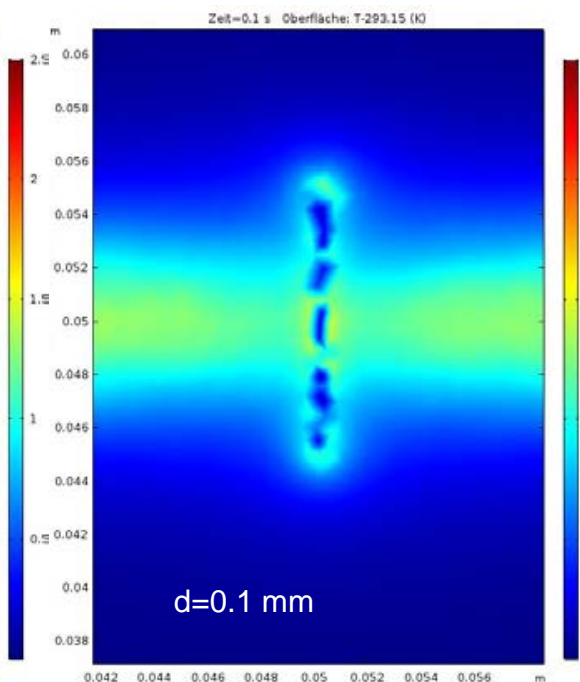

b)

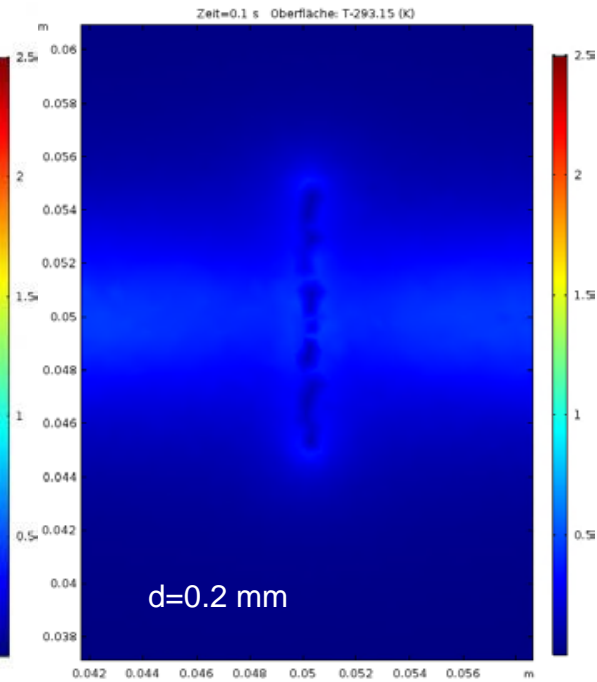

C)

Fig. 5: a) Calculated temperature distribution after $0.1 \mathrm{~s}$ of induction heating on the metal surface under the coating of $0.1 \mathrm{~mm}$ thickness. $b, c)$ temperature on top of the coating with a thickness of $0.1 \mathrm{~mm}$ and $0.2 \mathrm{~mm}$, respectively. The displayed temperature range is the same for a), b) and c)

The sound area under the wire is heated and shows the proximity effect (figure 5). Beside this, the typical crack patterns including the crack tip peaks can be recognized on the coating surface. However, the temperature contrasts are strongly attenuated with increasing coating thickness.

\section{Experiments}

Measurements were performed with two induction systems allowing for periodic and pulsed excitation.

A test block made of ferritic steel was prepared to contain artificial cracks of $5 \mathrm{~mm}$ length, $3 \mathrm{~mm}$ depth and $0.5 \mathrm{~mm}$ width (figure 6). Four of these cracks were investigated. One crack was on an uncoated area. The other cracks were under a black paint coating of $0.05 \mathrm{~mm}, 0.1 \mathrm{~mm}$ and $0.15 \mathrm{~mm}$ nominal paint thickness, respectively.

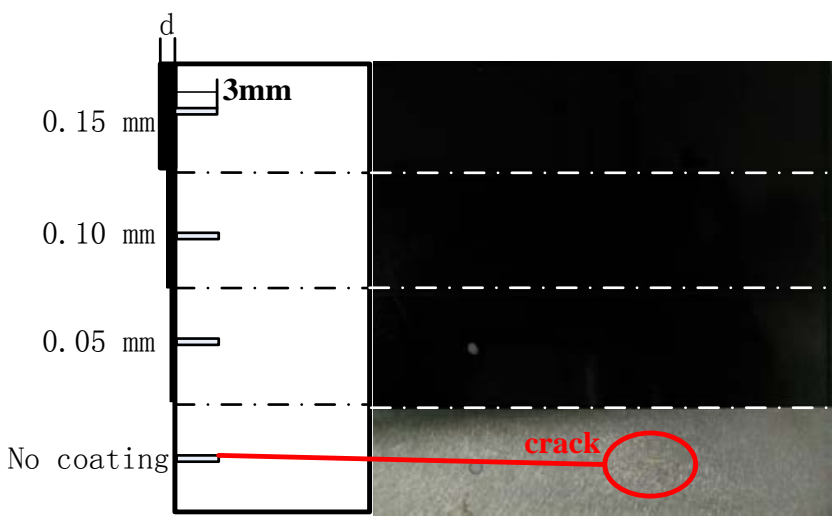

Fig. 6: Sketch of a painted steel block (left part: cross section, right part: photo from top with different coating thickness and artificial cracks

A second sample was constructed from layers of optically transparent adhesive tape (Tesafilm®), each $70 \mu \mathrm{m}$ thick. Such layers were applied over an artificial crack in ferritic steel with $10 \mathrm{~mm}$ length, $2.9 \mathrm{~mm}$ depth and $0.3 \mathrm{~mm}$ width. 


\subsection{Lock-in induction thermography}

The lock-in induction system used for these experiments consists of a function generator generating the induction frequency. It can be gated by the signal of a synchronisation box, which derives the modulation frequency from the camera frame clock, which was $146 \mathrm{~Hz}$. The signal from the function generator is amplified by a power amplifier with $200 \mathrm{~W}$ maximum output. A work-head consisting of a capacitor and the induction coil is operated at its resonance frequency of typically about $174 \mathrm{kHz}$. Figure 7 shows a photo of the lock-in induction system.

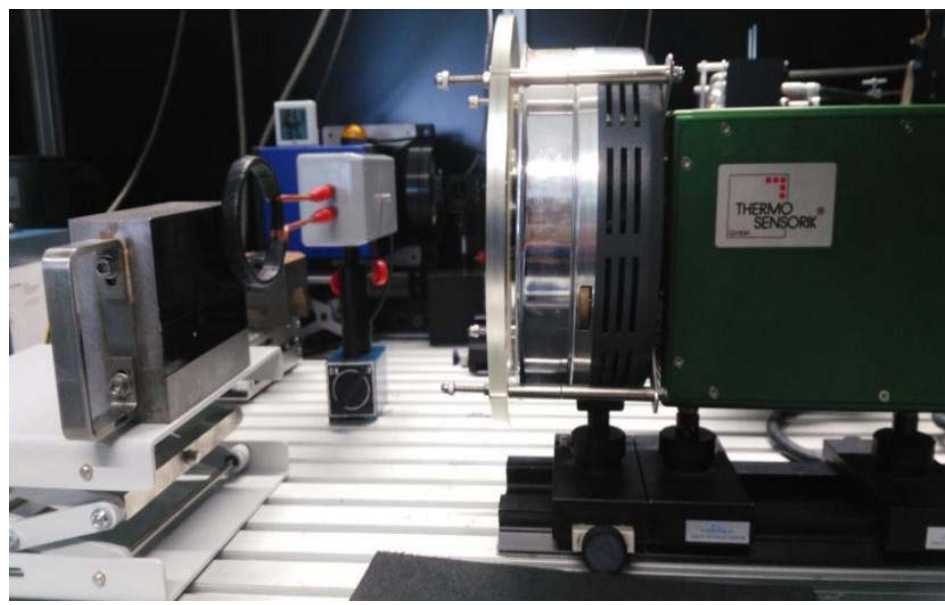

Fig. 7: Photo of the lock-in induction system

The cracks in the black painted test body were excited by an induction coil. After lock-in processing, thermal amplitude and phase images were obtained as shown in figure 8 . The coil was positioned to cross the crack. The general heating of the area under the coil as well as heating of the crack tips are visible. Along the profile lines in figure 8 , the amplitude and the phase contrast were determined for the different coating thickness.
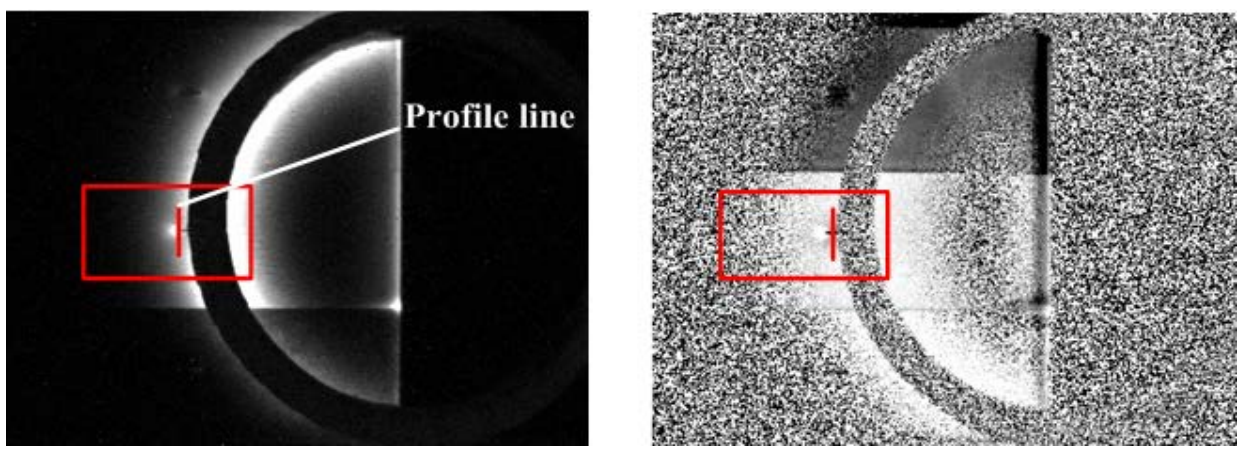

Fig. 8: Thermal amplitude image (left) and phase images (right) of a crack with $0.05 \mathrm{~mm}$ black paint coating at a modulation frequency $4.56 \mathrm{~Hz}$

In the following, the amplitude contrast was determined as the temperature difference between the peak signal and the signal from a reference area sufficiently away from the crack. The phase contrast was determined as the difference between the reference phase and the minimum of the phase at the crack. Figure 9 shows the thermal contrast due to the crack as a function of the coating thickness for both test bodies investigated. Both amplitude and phase contrast are decreasing with thickness, as can be expected from the analytical calculations. The adhesive layer coating shows a more rapid decrease of phase contrast with thickness than the black paint coating. This can be due to a different thermal diffusivity of the coating. Another reason is suggested by the measurement shown in figure 10. 


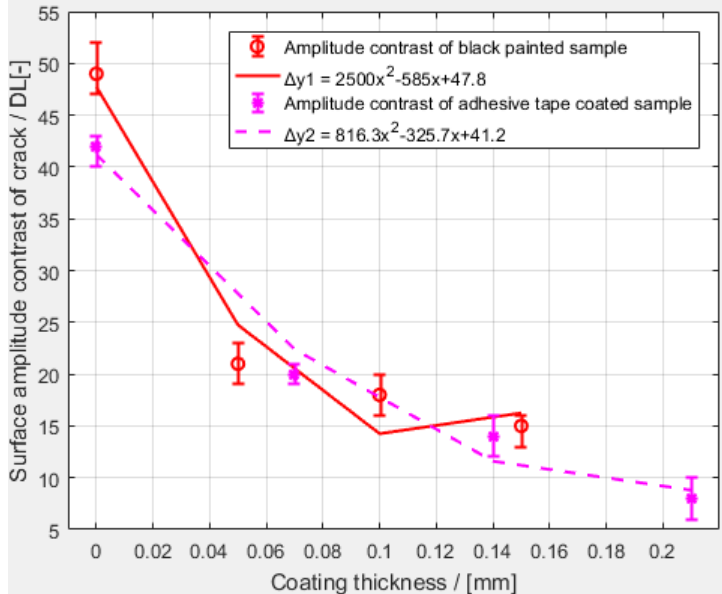

a)

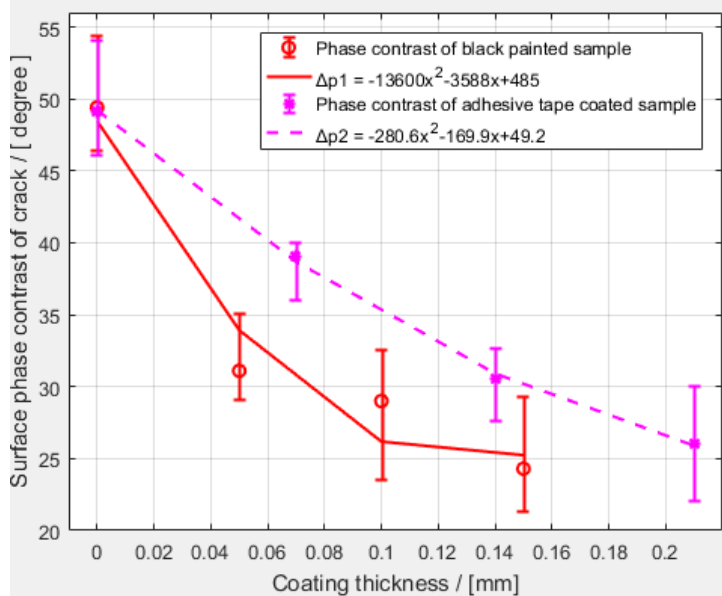

b)

Fig. 9: a) Thermal amplitude contrast of a crack under a black paint coating and an adhesive film coating, repectively, as a function of the coating thickness at a modulation frequency $4.56 \mathrm{~Hz}$. b) The corresponding phase image

The camera used for the lock-in experiments was a dual band IR camera with QWIP detector, which acquires two images simultaneously: One image is in the MWIR (mid-wave infrared, 4.4-5.2 $\mu \mathrm{m}$ ) band and one in the in the LWIR band (long-wave infrared, 7.8-8.8 $\mu \mathrm{m}$ ) [7]. The experimental results were usually analysed in the MWIR band. In figure 10 , the measured absolute amplitude crack contrast for the adhesive tape sample as a function of coating thickness is shown for both bands. The absolute crack contrast decreases with thickness (the estimated thermal diffusion length is $0.12 \mathrm{~mm}$ ). The decrease is more rapidly in the LWIR band than in the MWIR band. This is attributed to the coating transparency in the thermal infrared.

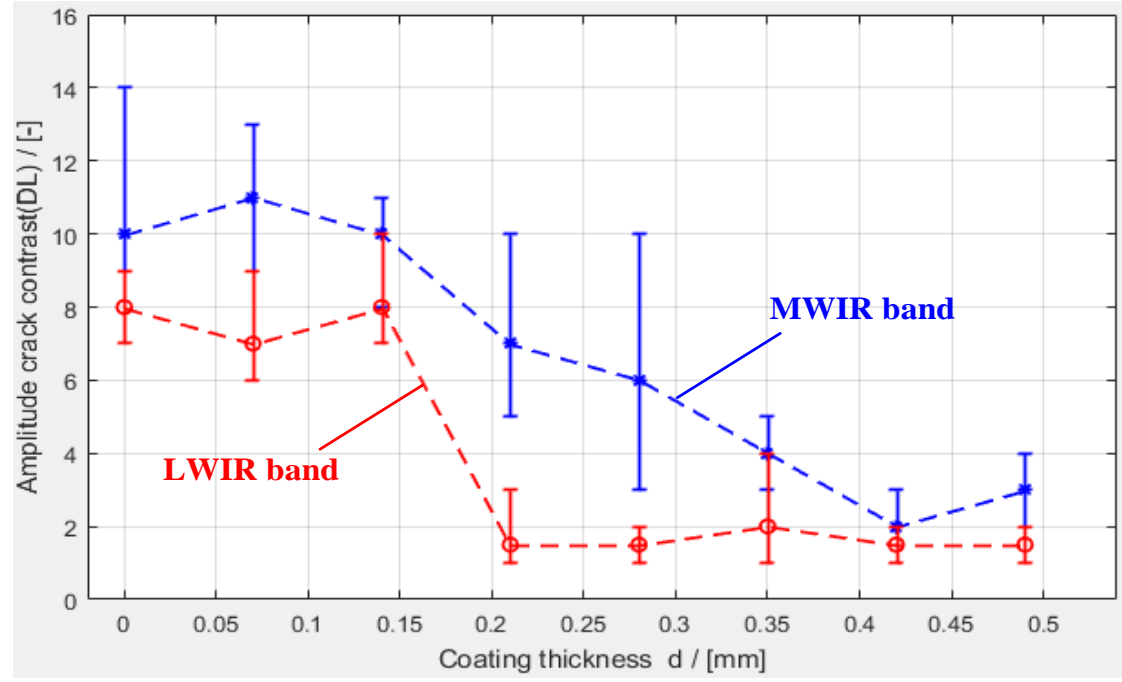

Fig. 10: Measured amplitude crack contrast under a coating of adhesive tape at a modulation frequency of $4.56 \mathrm{~Hz}$ as a function of the coating thickness detected in two different wavelength ranges

The assumption of the role of the transparency of the coating in the thermal infrared is supported by the infrared spectrum of the coating (figure 11). It was measured using an ExoScan mobile infrared spectrometer $\left(A_{2}\right.$ technologies) in a modified arrangement for transmission. From the spectrum one can see that the IR transmittance is much higher in the MWIR than in the LWIR band of the camera. 


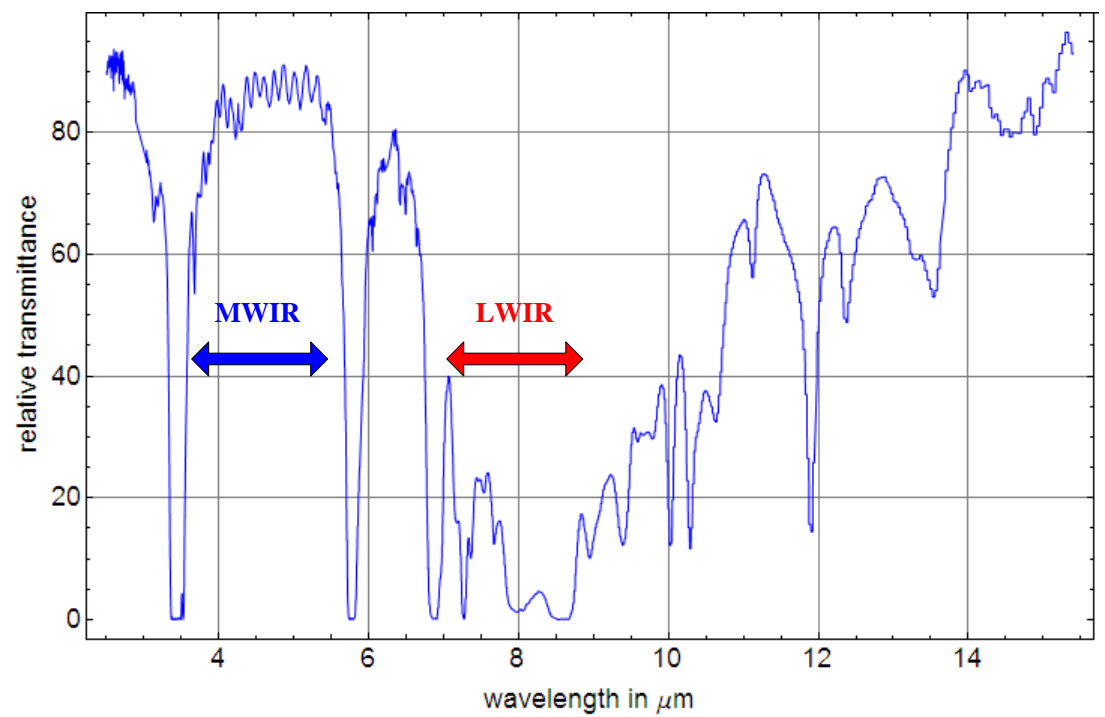

Fig. 11: Infrared transmission spectrum of one layer of adhesive tape. The sensitive bands of the dual-band camera used are indicated

\subsection{Pulsed induction thermography}

The pulsed induction system was based on an induction generator and workhead with a maximum output power of $10 \mathrm{~kW}$ (Hüttinger). A pancake-type coil was used with pulses of 100-200 ms duration (figure 12). The IR images were acquired using a FLIR SC5000 photodetector camera at a framerate of $170 \mathrm{~Hz}$. The pulse responses were recorded and then processed by a pulse phase thermography algorithm.

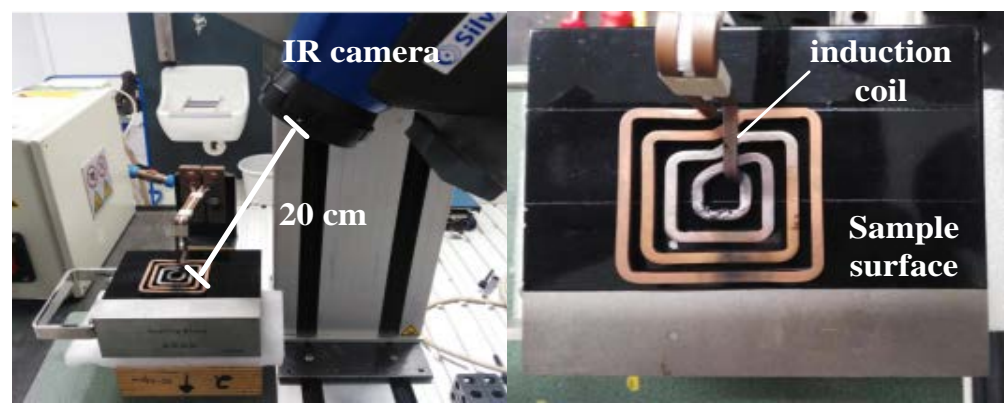

Fig. 12: Photo of the pulsed induction thermography system

In figure 13, a phase image of the black painted sample with artificial cracks is shown. The crack positions under the coatings are indicated by the three markers. The phase level of the sound areas is decreasing with increasing coating thickness. The crack contrast is weaker than the effect of the varying paint thickness. It is visualized by line scans over the cracks shown in the right part of figure 13.

Another parameter varied beside the coating thickness was the pulse duration of induction heating. Results are shown in figure 14. 


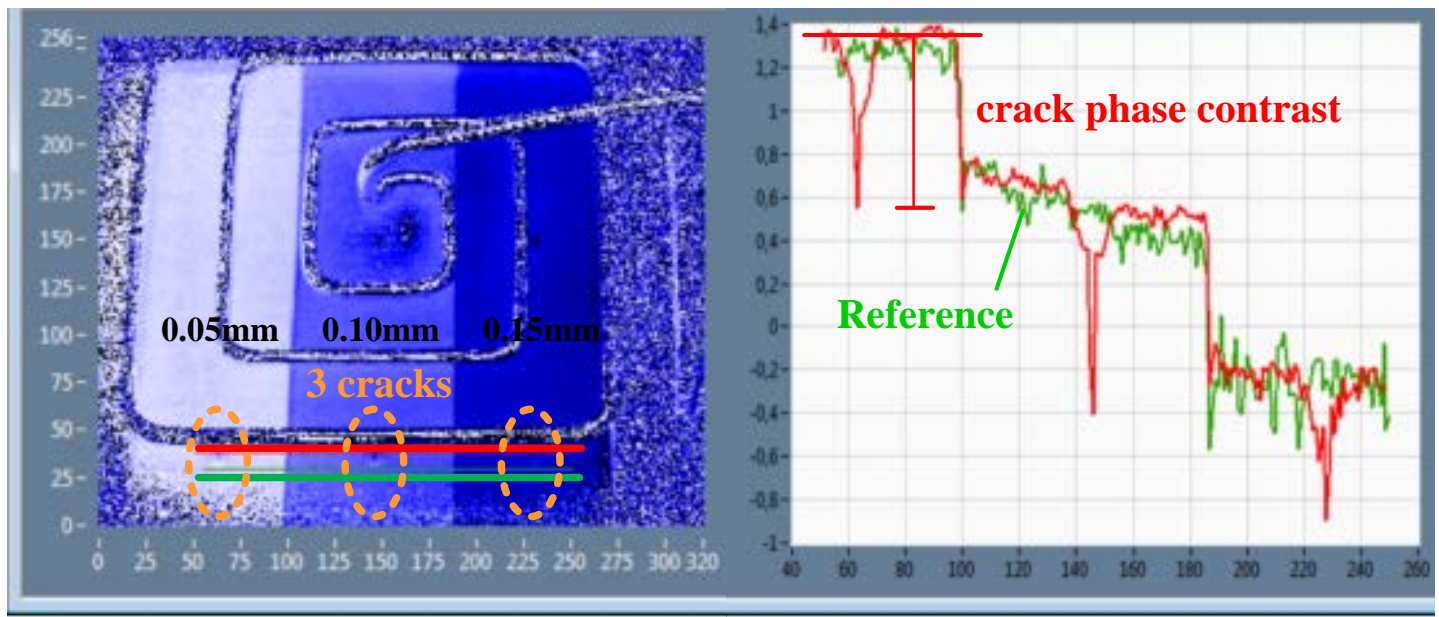

Fig. 13: Left: IR image of the sample with different paint thickness and a pancake-type coil.The red line crosses the cracks, the green line is a reference not affected by the crack contrast. Right: Phase signal profiles along the red and green lines

In figure 14, the measured phase contrast is shown as a function of the coating thickness. The phase contrast is larger for the shorter pulse duration, but for the longer pulses, the signal-to-noise of the contrast appears to be better.

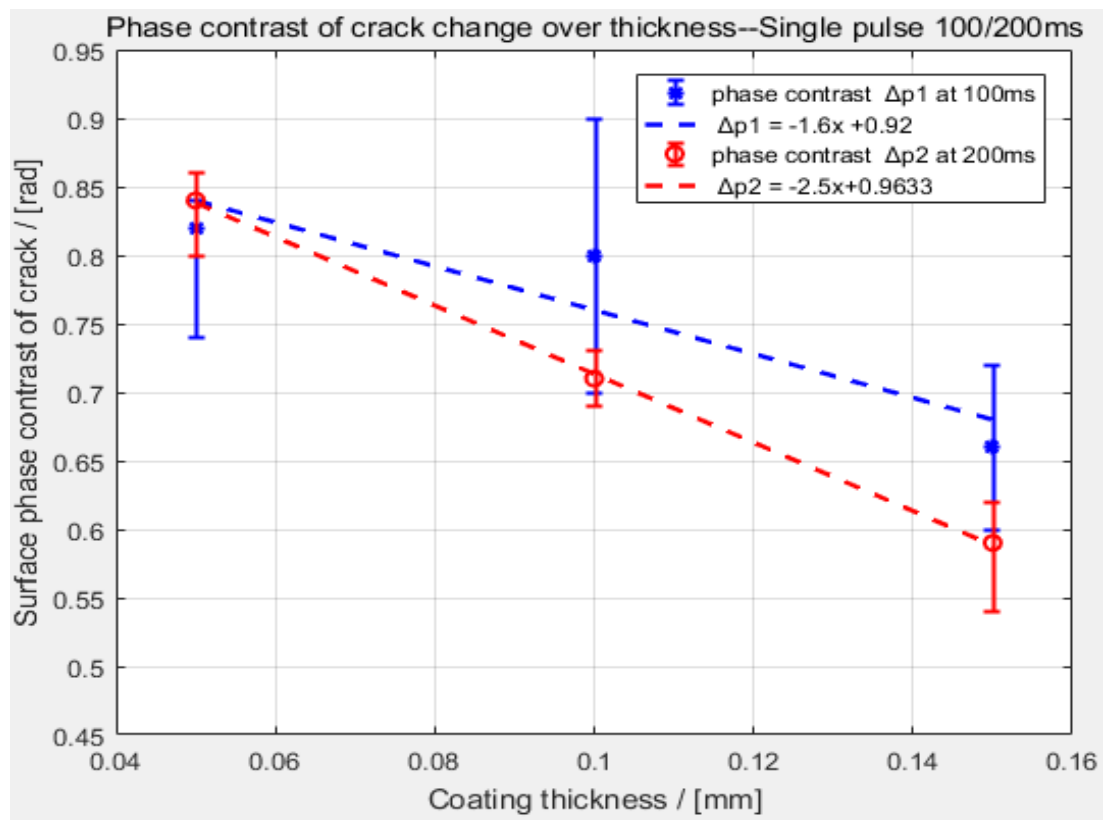

Fig. 14: Phase contrast for the crack as a function of the coating thickness at two different pulse lengths

\section{Conclusion}

The present calculations and experiments have confirmed that induction thermography for surface crack detection can be performed on polymer coated samples. The coating usually causes damping of the amplitude and phase contrast with increasing polymer thickness. About one to three thermal diffusion lengths can be penetrated. Thick polymer layers may require a few seconds to reach the maximum response. Then, the crack contrast at the metal surface may already be vanished.

The limitations given by the thermal diffusion length may be less strict, if the coating has a certain optical transparency in the thermal infrared. For polymer coatings, this was demonstrated experimentally by the dual-band measurements on the layers of adhesive tape. Further work will analyse this in a more quantitative way. 


\section{REFERENCES}

[1] Vrana J, PhD Thesis (in German) (Universität des Saarlandes, 2007) p. 106-107

[2] Frackowiak W, Bruchwald O, Zwoch Z, Reimche W, Maier H. Non-Destructive Damage Detection and Material Characterization of Turbine Components Using Megahertz Range Induction Thermography in Pulsed Mode, Proc. $19^{\text {th }}$ WCNDT, Munich 2016

[3] Xu C, Zhou N, Xie J, Gong X, Chen G. Investigation on eddy current pulsed thermography to detect hidden cracks on corroded metal surface. NDT\&E International 84 (2016) 27-35

[4] Yang R, He Y, Zhang H, Huang S. Through coating imaging and non-destructive visualization evaluation of early marine corrosion using electromagnetic induction thermography. Ocean Engineering 147 (2018) 277-288

[5] Li X, Liu Z, Jiang X, Lodewijks G. Method for detecting damage in carbon-fibre reinforced plastic-steel structures based on eddy-current pulsed thermography. Nondestructive Testing and Evaluation 33 (2018) 1-19

[6] Oswald-Tranta B. Induction Thermography for Surface Crack Detection and Depth Determination. Appl. Sciences (8) (2018) 257

[7] Abuhamad M and Netzelmann U. Dual-band active thermography on infrared transparent materials. Proc. 10th Int. Conf. on Quantitative Infrared Thermography (QIRT), 26.-30.7.2010, Quebec City, Canada, p. 868-875 